FLORIDA GEOLOGICAL SURVEY

$$
\begin{aligned}
& 1951
\end{aligned}
$$

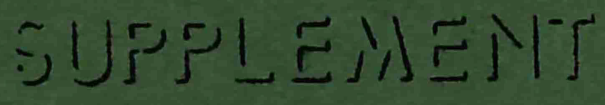

$$
\begin{aligned}
& \text { (1) }
\end{aligned}
$$

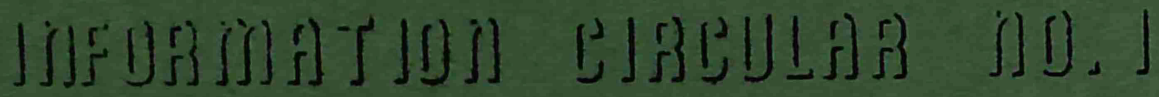

$$
\begin{aligned}
& \text { ( } f 35 y] \text { J }
\end{aligned}
$$

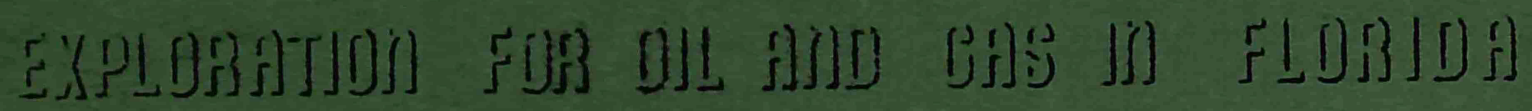

BY

HERMAN GUNTER, DIRECTOR

TALLAHASSEE, FLORIDA

JANUARY I, 1952 



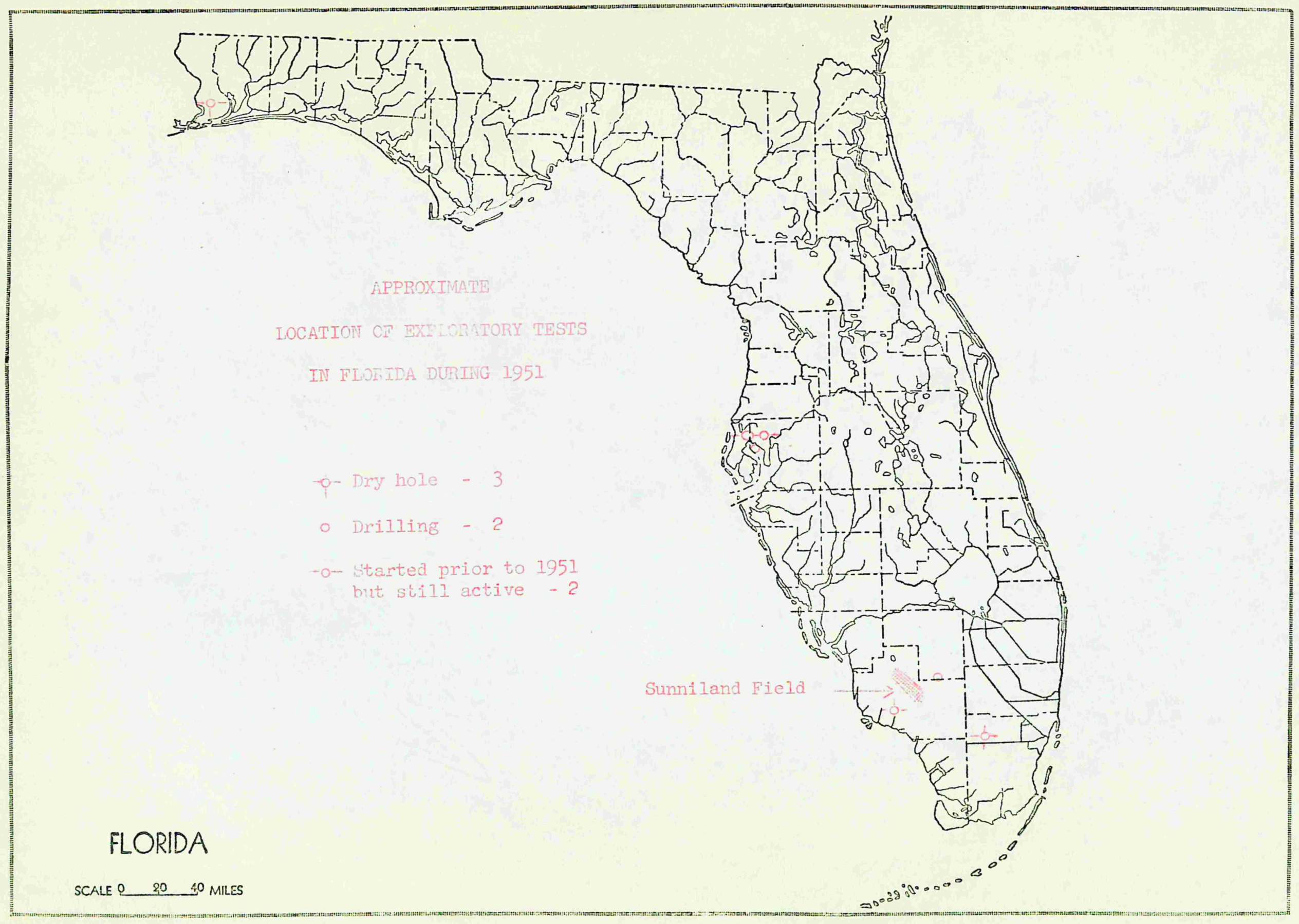


FLORIDA GEOLOGICAL SURVEY

$$
\begin{aligned}
& 1951
\end{aligned}
$$

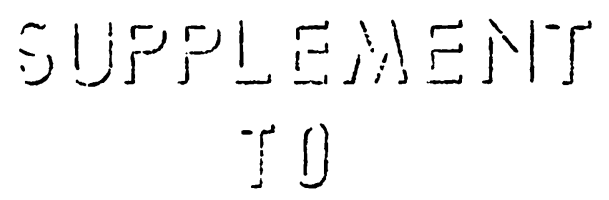

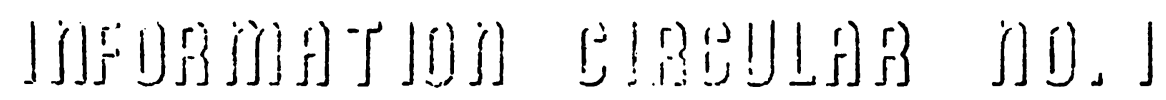

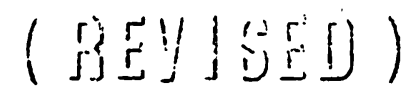

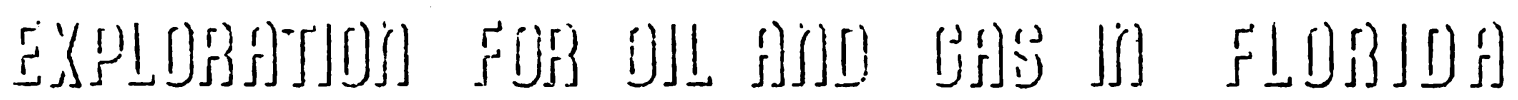
$B Y$

HERMAN GUNTER. DIRECTOR

TALLAHASSEE, FLORIDA

JANUARY 1.1952 
1951 SUPPLEMENT TO

INFORMATION CIRCULAR NO. 1 - REVISED

EXPLORATION FOR OIL AND GAS IN FLORIDA

By

Herman Gunter, Director

FLORIDA GEOLOGICAI SURVEY

Exploratory activities in Florida during 1951 continued the downward trend begun in 1950. In fact, only five tests were drilled, one of which was the deepening of the William G. Blanchard's Everglades No. 1 (shut down since 1944) on the Tamiami Trail, about 43 miles west of Miami, by the McCord Oil Corporation. Three of these tests were completed and abandoned as dry holes within the year. Two were drilling on December 31 - the Humble $0 i 1$ and Refining Company's Collier Corporation "B" No. I, Hendry County, at about 9,500 feet, and the R. E. Skinner's J. A. Boyd Estate No. 1, Pinellas County, at about 1,700 Peet. Incomplete at the end of the year are two other wells in Pinellas County, one begun in 1945, the other in 1950, by R. E. Skinner.

In 1951, exploratory drilling of special significance includes the operation of the McCord 011 Corporation in deepening the old Everglades No. I (renamed the Damoco Oil Corporation, et al, No. I) to the"Sunniland lime" in which encouraging shows of oil were found. The information obtained resulted in additional geophysical prospecting which it is reported. will bring about further test drilling in the area. Also, 
during October-December, 1951, the Commonwealth Oil Company drilled the Marcus Ilschkoff, et al, No. I, bordering the Perdido Bay, Escambia County, extreme western Florida, which reached a total depth of 7,494 feet. Earlier tests were drilled in southern Escambia County, Alabama, one of which yielded significant shows of oil, and these have centered new interest in northwestern Florida. Without doubt there will be further drilling activity and development in this part of the State. 
SUMMARY OF EXPLORATION ACTIVITIES DURTNG 1951

(For details see tables under respective counties)

WELIS BEGUN AND COMPLETED IN 1951

COLLIER COUNTY

Humble 011 and Refining Company

Collier Corporation No. 1, $4 \mathrm{ml}$. N. Belle Meade, 12,600 ft. D \& A

DADE COUNTY

McCord Oil Corporation

Damoco Oil Development Company, et al, No. I (formerly, Wm. G. Blanchard, Everglades No. 1), $43 \mathrm{mi}$. W. Miami, deepened from 10,281 ft. to $11,885 \mathrm{ft}$. Oil shows, good bleeding cores recorded, but could not develop into commercial production.

\section{ESCAMBIA COUNTY}

Commonwealth 011 Company

Marcus Lischkoff, et al, No. I, about $3 \mathrm{ml}$. NW of Myrtle Grove, 7,494 ft. D \& A

WELIS DRILIING ON DECEMBER 31, 1951

HENDRY COUNTY

Humble Oil and Refining Company

Collier Corporation " $\mathrm{B}$ " No. 1 , about $14 \mathrm{mi}$. SE of Immokalee. Coring at about $9,500 \mathrm{ft}$. on December 31, 1951

PINELLAS COUNTY

R. E. Skinner

J. A. Boyd Estate No. 1, about $8 \mathrm{ml}$. SE of Tarpon Springs. Drilling at about 1,700 ft. on December 31, 1951 . 
WELLS BEGUN PREVIOUS TO 1951 AND NOT COMPLETED

DECEMBER 31, 1951

PINELIAS COUNTY

R. E. Skinner

J. A. Boyd, Block 7, No. 1, about $6 \mathrm{ml}$. SE of Tarpon Springs, begun May 2, 1945. Depth 3,563 ft. Fishing for drill bit.

J. A. Boyd, Block 10 and 4, No. 1, about $6 \mathrm{mi}$. SE of Tarpon Springs, begun July 10, 1950. Last depth reported 1,248 ft. Operations temporarily suspended, awaiting rig.

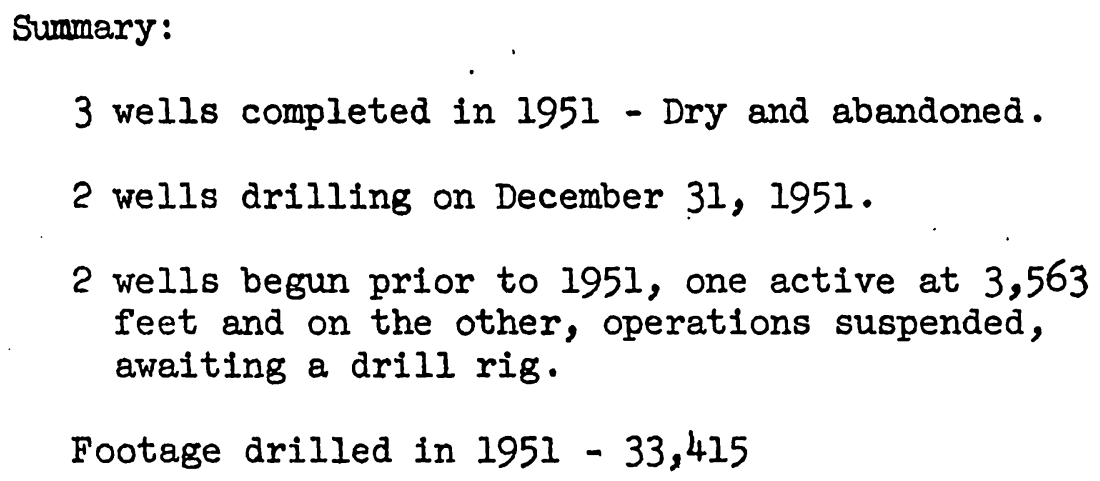

During 1951, test wells were drilled in flve counties - Collier, Dade, Escambia, Hendry and Pinellas. For details on these see the tables on pages 7 through 11 . 
OII PRODUCTION IN 1951

Oil production from the Sunniland Field in Collier County, so far the only field in Florida, totaled 596,043 barrels in 1951. This is an increase of 110,022 barrels over the 1950 production. The crude oil is transported by tank truck from the field to Port Everglades at Fort Lauderdale. Table No. 1 on the following page gives the monthly, yearly and cumulative production since the discovery well came in on September 26, 1943.

PROSPECTS FOR 1952

The encouraging shows of oil in the McCord Oil Corporation well on the Tarniami Trail undoubtedly strengthens the potential prospect in that area. It is not too much to predict that additional development will occur in that region.

Interest, too, has been turned to northwestern Florida because of the shows of oil encountered in the Stanolind Oil and Gas Company F. A. Steward No. I, Escambia County, Alabama, further exploratory activity in the area is assured. Escambia County, Alabama, adjoins Escambia and Santa Rosa counties, Florida, and this well is located approximately five miles north of the Florida State line. The Humble 011 and Refining Company has made a location on the A. W. Moye farm, located about one and one-half miles east, and one-quarter mile south of the Stanolind well, which is indicative of the importance of the find. 
Table 1

PRODUCTION OF OIL FROM HUMBLE OIL \& REFINING COMPANY'S SUNNILAND FIELD COLLIER COUNTY, FROM SEPT. 26, 1943, THROUGH DECEMBER 31, 1952

\begin{tabular}{|c|c|c|c|c|c|c|c|c|c|}
\hline Month & 1943 & 1944 & 1945 & 1946 & 1947 & 1948 & 1949 & 1950 & 1951 \\
\hline Jan. & & 2108 & 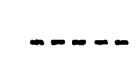 & 3899 & 7464 & 25149 & 37695 & 32095 & 47845 \\
\hline Feb. & & 1404 & 402 & 3454 & 5203 & 22853 & 32617 & 26091 & 43543 \\
\hline Narch & & 1100 & 581 & 2982 & 18795 & 25688 & 38880 & 31963 & 48613 \\
\hline April & & 1054 & 403 & 4151 & 16362 & 23597 & 42859 & 31136 & 49990 \\
\hline May & & 1115 & 725 & 6776 & 20440 & 21266 & 39756 & 28146 & 49288 \\
\hline June & & 822 & 4110 & 7676 & 31065 & $24+092$ & 39956 & 47649 & 49823 \\
\hline July & & 1.123 & 5700 & 6450 & 31395 & 25543 & 44070 & 46740 & 54499 \\
\hline Aug. & & 957 & 4455 & 4160 & 31021 & 24011 & 44764 & 50578 & 50294 \\
\hline Sept. & $\begin{array}{l}\text { Discov. } \\
\text { Date } \\
9 / 26 / 43 \\
\end{array}$ & 516 & 2775 & 6544 & 28431 & 22475 & 31437 & 48130 & 49609 \\
\hline oct. & 648 & 609 & 2976 & $\ldots$ & 22445 & 27003 & 28634 & 45715 & 51725 \\
\hline Nov. & 643 & 581 & 2666 & 2.408 & 23948 & 20331 & 31054 & 52813 & 48008 \\
\hline Dec. & 2741 & 449 & 2717 & 8384 & 22776 & 29213 & 29998 & 44965 & 52806 \\
\hline $\begin{array}{l}\text { TOTAL } \\
\text { BBLS. } \\
\end{array}$ & 4032 & 1.1838 & 27510 & 56884 & 259345 & 291221 & 441.720 & 486021 & 596043 \\
\hline $\begin{array}{l}\text { TOTAL } \\
\text { CURIULA- } \\
\text { TIVE PROD. } \\
\text { BARRELS } \\
\end{array}$ & 4032 & 15870 & 43380 & 100264 & 359619 & 650830 & 1092550 & 1578571 & 2174614 \\
\hline
\end{tabular}


COLLIER COUNTY

\begin{tabular}{|c|c|c|c|c|c|c|c|}
\hline $\begin{array}{l}\text { FGS } \\
\text {-NO. }\end{array}$ & COMPANY OY OWNERS & $\begin{array}{l}\text { FARM or } \\
\text { WELL NAME }\end{array}$ & LOCATION & $\begin{array}{c}\text { ELEVA- } \\
\text { TION }\end{array}$ & $\begin{array}{l}\text { YEAR } \\
\text { COM- } \\
\text { PLETED }\end{array}$ & DEPTH & REMARKS \\
\hline $\begin{array}{l}W- \\
2420\end{array}$ & $\begin{array}{l}\text { Humble 0il and } \\
\text { Refining Company } \\
\text { P. 0. Box } 2180 \text {, } \\
\text { Houston } 1 \text {, Texas }\end{array}$ & $\begin{array}{l}\text { Collier Corporation } \\
\text { No. } 1 \\
\text { Permit No. } 130\end{array}$ & $\begin{array}{l}1884.5^{\prime} \text { from N/L } \\
\& 2150.3^{\prime} \text { from } \\
\text { W/L of Sec. } 27 \text {, } \\
\text { T50S, R26E } \\
\text { Belle Meade, a- } \\
\text { bout } 4 \text { mi. N of }\end{array}$ & $25^{\prime} \mathrm{DF}$ & 1951 & 12,600 & $\begin{array}{l}\text { Dry and abandoned. Form } 2 \\
\text { appvd April } 24,1951 \text {. Form } \\
5 \text { appvd Sept. } 14,1951 \text {. } 83 \\
\text { samples - cuttings:0-2560' } \\
\text { Continuous diamond cores: } \\
4891 \text { to } 5900 \text { '; and } 7710- \\
12600 \text { '. Electric 10g:1025- } \\
12516 \text { '. Driller's log: } \\
0-12600^{\prime} \text {. }\end{array}$ \\
\hline
\end{tabular}


DADE COUNTY

\begin{tabular}{|c|c|c|c|c|c|c|c|}
\hline $\begin{array}{l}\text { FGS } \\
\text { NO. }\end{array}$ & COMPANY OR OWNERS & $\begin{array}{l}\text { FARM or } \\
\text { WELL NAME }\end{array}$ & LOCATION & $\begin{array}{l}\text { ELEVA- } \\
\text { TION }\end{array}$ & $\begin{array}{l}\text { YEAR } \\
\text { COM- } \\
\text { PLETED } \\
\end{array}$ & DEPTH & REMARKS \\
\hline w- & $\begin{array}{l}\text { McCord Oil Corp. } \\
\text { J. L. McCord, Pres. } \\
615 \text { s/W 2nd Ave., } \\
\text { Miami, Florida }\end{array}$ & $\begin{array}{l}\text { Damoco Oil De- } \\
\text { velopment Co., } \\
\text { et al, No. I } \\
\text { (Formerly the } \\
\text { Wm. G. Blanchard } \\
\text { Everglades No. I) } \\
\text { Permit No. } 129\end{array}$ & $\begin{array}{l}200^{\prime} \mathrm{E} \& 100^{\prime} \mathrm{N} \\
\text { of SW/cor. of Sec. } \\
\text { 31, T535, R.35E. } \\
\text { ahout } 1.3 \mathrm{mil} . \mathrm{W} \text { of } \\
\text { Miamt }\end{array}$ & $17^{\prime} \mathrm{DF}$ & 1951 & $11,885^{*}$ & $\begin{array}{l}\text { Form } 2 \text { appvd Jan. } 28,1951 \text {, } \\
\text { to deepen from 10,281 ft. } \\
\text { Form } 5 \text { appvd May } 22,1951 \text {. } \\
\text { Shows of oil in "Sunniland } \\
\text { lime" zone. } 3 \text { drill stem } \\
\text { tests attempted. D \& A. } \\
885 \text { samples: 0 to } 10284 \text { ft. } \\
\text { Continuous wire line cores: } \\
7415-10284^{\prime} \text {. Black oil } \\
\text { shows recorded Irom } 9445^{\prime} \\
\text { and lower depths. } \\
\text { Deepening: Continuous dia- } \\
\text { mond cores: 10281-11885' } \\
\text { Electric log: 324l-11806'. } \\
\text { Description of cores, } \\
\text { lithologic log corrected } \\
\text { to vertical, drill stem } \\
\text { tests, coring and time log. }\end{array}$ \\
\hline
\end{tabular}


ESCAMBIA COUNTY

\begin{tabular}{|c|c|c|c|c|c|c|c|}
\hline $\begin{array}{l}\text { FGS } \\
\text { NO. }\end{array}$ & COMPANY or OWNERS & $\begin{array}{l}\text { FARM or } \\
\text { WELL NAME }\end{array}$ & LOCATION & $\begin{array}{l}\text { ELEVA- } \\
\text { TION }\end{array}$ & $\begin{array}{l}\text { YEAR } \\
\text { COM- } \\
\text { PLETED }\end{array}$ & DEPTH & REMARKS \\
\hline $\begin{array}{l}\text { W- } \\
2519\end{array}$ & $\begin{array}{l}\text { Commonwealth 0il } \\
\text { Company } \\
615 \text { S/W 2nd Ave., } \\
\text { Miami, Florida } \\
\text { J.L.McCord, } \\
\text { V. Pres. }\end{array}$ & $\begin{array}{l}\text { Marcus Lischkoff, } \\
\text { et al, No. } 1 \\
\text { Permit No. } 132\end{array}$ & $\begin{array}{l}\text { Cen. SW } / 4 \mathrm{NW} / 4 \\
\text { Sec. } 8, \mathrm{~T} 2 \mathrm{~S}, \mathrm{R} 3 \mathrm{lW} \text {, } \\
\text { about } 3 \mathrm{mi} \text {. NW } \\
\text { of Myrtle Grove }\end{array}$ & $19^{\prime} \mathrm{DF}$ & 1951 & 7,494 & 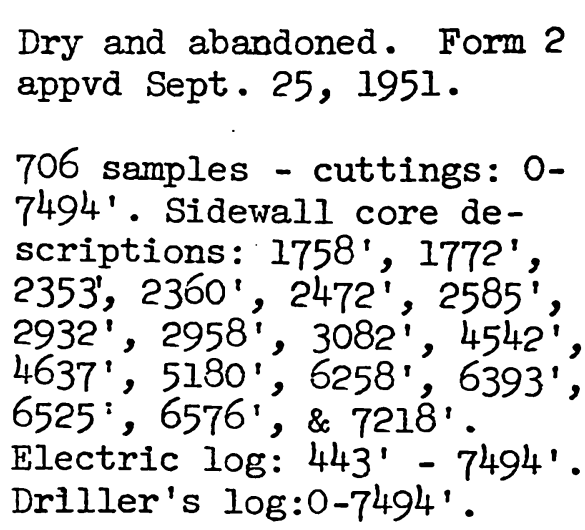 \\
\hline
\end{tabular}


HENDRY COUNTY

\begin{tabular}{|c|c|c|c|c|c|c|c|}
\hline $\begin{array}{l}\text { FGS } \\
\text { NO. }\end{array}$ & COMPANY OY OWNERS & $\begin{array}{l}\text { FARM or } \\
\text { WELL NAME }\end{array}$ & LOCATION & $\begin{array}{c}\text { ELEVA- } \\
\text { TION }\end{array}$ & $\begin{array}{l}\text { YEAR } \\
\text { COM- } \\
\text { PLETED }\end{array}$ & DEPTH & REMARKS \\
\hline W- & $\begin{array}{l}\text { Humble 0il and } \\
\text { Refining Company } \\
\text { P.O.Box } 2180 \\
\text { Houston, Texas }\end{array}$ & $\begin{array}{l}\text { Collier Corp., } \\
\text { "B" No. } 1 \\
\text { Permit No. } 133\end{array}$ & $\begin{array}{l}2161.7^{\prime} \text { from } \mathrm{S} / \mathrm{L} \\
1863.5^{\prime} \text { from } \mathrm{E} / \mathrm{L} \\
\mathrm{Sec} .14, \mathrm{~T} 4 \mathrm{TS}, \mathrm{R} 31 \mathrm{E} \\
\text { About } 14 \mathrm{mi} \text {. S/E } \\
\text { of Tmmokalee }\end{array}$ & $40^{\prime} \mathrm{DF}$ & & & $\begin{array}{l}\text { Form } 2 \text { apprd. Oct. 2,1951. } \\
\text { Corjns at about } 9,500 \mathrm{ft} . \text {, } \\
\text { Lecember } 31 \text {, } 1951 .\end{array}$ \\
\hline
\end{tabular}


PINELIAS COUNTY

\begin{tabular}{|c|c|c|c|c|c|c|c|}
\hline $\begin{array}{l}\text { FGS } \\
\text { NO. }\end{array}$ & COMPANY or OWNERS & $\begin{array}{l}\text { FARM or } \\
\text { WELL NAME }\end{array}$ & LOCATION & $\begin{array}{l}\text { ELEVA- } \\
\text { TION }\end{array}$ & $\begin{array}{l}\text { YEAR } \\
\text { COM- } \\
\text { PLETED }\end{array}$ & DEPTH & REMARKS \\
\hline W- & $\begin{array}{l}\text { R. E. Skinner } \\
\text { P.0.Box } 2939 \\
\text { Pampa 1, Florida. }\end{array}$ & $\begin{array}{l}\text { J. A. Boyd } \\
\text { Fistate No. } 1 \\
\text { Permit No. } 3.31\end{array}$ & $\begin{array}{l}500.5^{\prime} \mathrm{N} \text { and } 167^{\prime} \mathrm{W} \\
\text { of } \mathrm{SE} / \mathrm{cor} . \text { of } \mathrm{SE} / 4 \\
\text { of } \mathrm{NW} / 4 \text { of } \mathrm{Sec} .1 \text {, } \\
\mathrm{T} 28 \mathrm{~S}, \mathrm{Rl} 6 \mathrm{E} \text {, eight } \\
\text { mi.S/E of Tarpon } \\
\text { Springs. }\end{array}$ & $22^{\prime} \mathrm{Grd}$ & . & & $\begin{array}{l}\text { Form } 2 \text { appvd July } 3,1951 \\
\text { Drilling at about 1,700' } \\
\text { on December 31, 1951. }\end{array}$ \\
\hline
\end{tabular}



